\title{
Relationship between Ambulatory Pulse Pressure, Pulse Pressure In- dex and Coronary Artery Disease in Patients with Hypertension
}

\author{
Jiajuan Chen, Yunjie Wei, Zhen Gao, Haijun Xu, Hezhong Zhu* \\ Taihe Hospital, Affiliated Taihe Hospital of Hubei University of Medicine, Shiyan 442000, Hubei province, China. \\ E-mail: 769301593@qq.com
}

\begin{abstract}
Objective: To analyze the relationship between ambulatory pulse pressure, pulse pressure index, and coronary artery disease in patients with hypertension. Methods: From February 2018 to February 2019, a group of 100 patients with hypertension (control group) and a group of 100 patients with hypertension and coronary artery disease (experimental group) were selected to monitor and analyze dynamic pulse pressure and pulse pressure indicators. Results: In terms of clinical indicators, values of NPPI, 24hPP and 24hPPI in the experimental group were significantly higher than those in the control group. $\mathrm{P}<0.05$ indicates that there is statistical value in the data difference. Conclusion: In the clinical diagnosis of hypertension patients, ambulatory pulse pressure, pulse pressure index are highly correlated with the risk of coronary artery disease. Therefore, researchers should actively pay attention to the relevant indicators of patients to lay a solid foundation for the effective protection of patients' health.
\end{abstract}

Keywords: Hypertension; Ambulatory Pulse Pressure; Pulse Pressure Index; Coronary Artery Disease

\section{Introduction}

With the increasing trend of population aging in recent years, the incidence of various cardiovascular diseases has been on the rise, causing harm and threats to people's health. On this issue, some medical researchers pointed out that there is a close correlation between hypertension and coronary heart disease after analyzing research data. Based on this, medical researchers have conducted an in-depth analysis of the correlation between pulse pressure of hypertension patients and coronary heart disease in different periods in order to effectively protect the health of patients with hypertension, thus further realizing a reasonable analysis of health problems and providing help for the prevention of coronary heart disease for hypertension patients. After ana- lyzing a large number of data, researchers pointed out that pulse pressure, as one of the important clinical indexes of human body, is regarded as an important index in the diagnosis of cardiovascular diseases. Therefore, in order to further analyze the relationship between hypertension and coronary heart disease, medical researchers have made studies on pulse pressure of hypertension patients in different periods based on relevant clinical data, aiming at further exploring the relationship between pulse pressure and coronary heart disease, and thus to help hypertensive patients realize reasonable prevention of coronary heart disease effectively. This article explores and analyzes the relevant contents which will be reported as follows.

Copyright (C) 2021 Jiajuan Chen et al.

doi: 10.18686/aem.v10i1.187

This is an open-access article distributed under the terms of the Creative Commons Attribution Non-Commercial License

(http://creativecommons.org/licenses/by-nc/4.0/), which permits unrestricted non-commercial use, distribution, and reproduction in any medium,

provided the original work is properly cited. 


\section{Materials and methods}

\subsection{General information}

From February 2018 to February 2019, 100 patients with hypertension (control group) and 100 patients with hypertension and coronary heart disease (experimental group) in our hospital were selected as the research samples. There were 60 males and 40 females in the control group, aging from 35 to 71 years old. There were 61 males and 39 females in the experimental group, aging from 37 to 74 years old. The basic data has no statistical value $(\mathrm{P}>0.05)$.

\subsection{Methods}

All patients received ambulatory blood pressure monitor for their blood pressure indicators, and the monitor equipment is Spacelabs90217. All patients took safety test of related equipment between 8: 00 and 10: 00 a.m. In this process, medical staff helped patients to tie the cuff of the equipment to the left upper arm and adjust the parameters of the equipment to ensure that the reasonable measurement of the patient's pulse pressure index can be effectively collected during the period from 6 : 00 to 22: 00 and from 22: 00 to 6: 00 of the next day, providing corresponding data for the development and implementation of the follow-up research work. During blood pressure dynamic monitoring, medical staff should guide patients to avoid strenuous exercise to effectively realize the reasonable measurement of accurate blood pressure value, which provides guarantee for the next progress of monitoring work. At the same time, medical personnel should actively inform the monitoring instructions to ensure that patients do not use electronic devices, such as mobile phones, computers and tablets, in order to avoid the influence of electronic products on equipment during ambulatory blood pressure monitoring. In this period, medical personnel should actively and reasonably answer the related questions raised by patients, and effectively solve patient's questions, so as to lay a solid foundation and guarantee for the smooth development of the clinical diagnosis and treatment.

\subsection{Observation indicators}

In this study, the patients' night pulse pressure index (NPPI), 24-hour pulse pressure (24hPP) and 24-hour dynamic pulse pressure index (24hPPI) were taken as important monitoring indexes. Moreover, medical researchers should compare and analyze the ROC curves of patients with different indexes to provide corresponding guidance for the follow-up research work.

\subsection{Statistical method}

In this study, the statistical software SPSS20.0 is selected for data calculation, in which ( $\mathrm{x} \overline{\mathrm{s}}$ ) stands for measurement data, $\mathrm{t}$ test $\%$ stands for counting data, and $\mathrm{X}^{2}$ test. For data differences, $\mathrm{p}<0.05$ indicates that there is statistical value.

\section{Results}

\subsection{Comparisons of monitoring data}

In terms of clinical indicators, the values of NPPI, 24hPP and 24hPPI in the experimental group are significantly higher than those in the control group. $\mathrm{P}<0.05$ indicates that there is statistical value. Detailed data refer to Table 1.

Table 1. Pulse pressure and pulse pressure index of patient

\begin{tabular}{lllll}
\hline Group & $\mathbf{n}$ & NPPI & 24Hpp (mmHg) & 24hPPI \\
\hline Experimental group & 100 & $0.45 \pm 0.08$ & $50.57 \pm 1.43$ & $0.42 \pm 0.03$ \\
Control group & 100 & $0.37 \pm 0.06$ & $46.01 \pm 1.51$ & $0.38 \pm 0.02$ \\
t & - & 8.000 & 21.927 & 11.094 \\
$\mathrm{P}$ & - & $<0.05$ & $<0.05$ & $<0.05$ \\
\hline
\end{tabular}

\subsection{Logistic regression model analysis}

According to the analysis of ROC curve, areas under ROC curve of NPPI and 24hPPI in hypertension pa- tients with coronary heart disease are 0.68 and 0.66 respectively, larger than those under ROC curve of $24 \mathrm{~h}$ PP. Related figure is presented as follows. 


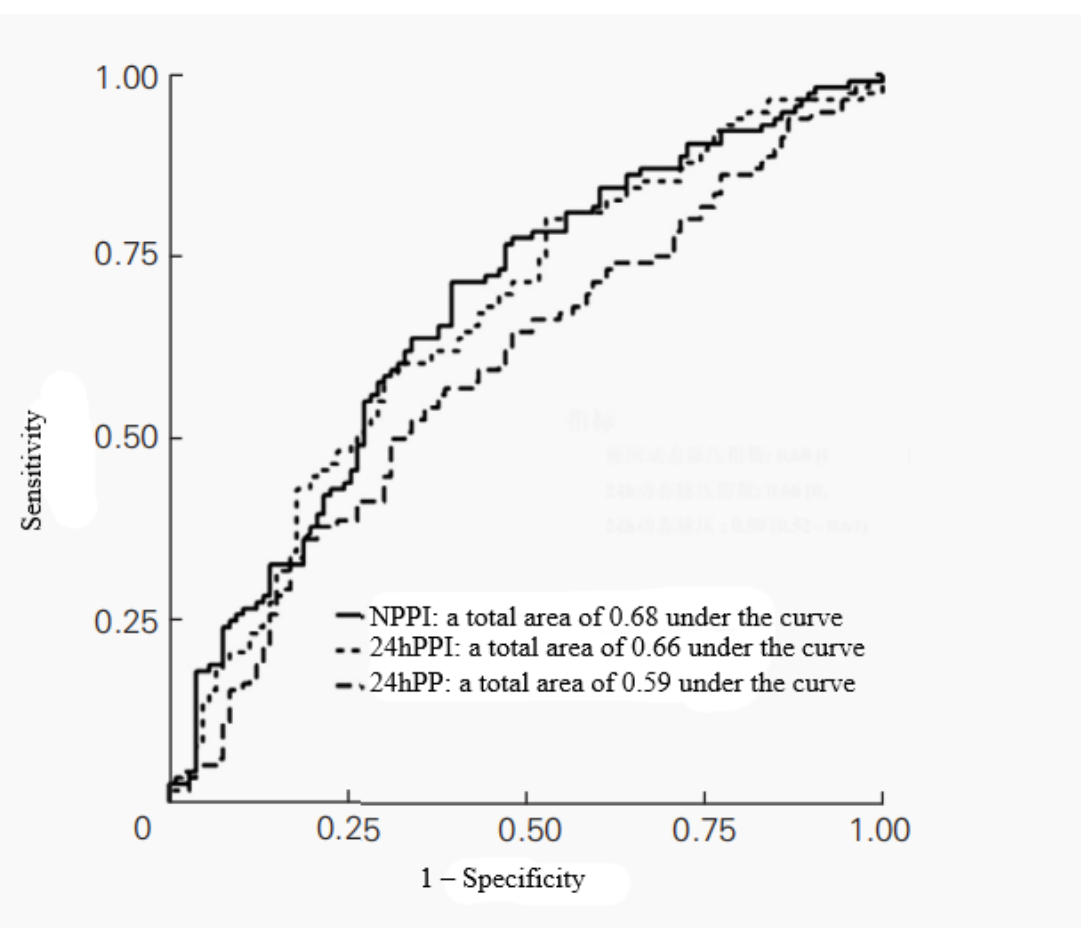

Figure 1. ROC curve.

\section{Discussion}

In clinical process, hypertension patients with coronary heart disease, are common in clinic treatment. Medical researchers pointed out that such situation often do extremely harm to patients' health, which will make the treatment more difficult and complicated. Particularly, as one of common diseases, hypertension can seriously affect the blood pressure index of patients, which leads to a significant increase in systolic blood pressure and diastolic blood pressure. If it cannot be controlled effectively in time, the excessive blood pressure will cause huge extra burden on the blood vessels of patients. It will also lead to an increasing trend of cardiovascular accidents and increasing the risk of coronary heart disease, which are extremely unfavorable to the health of patients. On this issue, researchers analyzed a large number of data and pointed out that the pulse pressure index of hypertension patients is relatively high, which can also be used as a predictor of coronary heart disease patients. Therefore, medical researchers should actively pay reasonable attention to the pulse pressure and pulse pressure index of hypertension patients, in order to effectively realize the reasonable prevention of coronary heart disease in hypertension patients in the nursing process. In such cases, they can analyze the probability of coronary heart disease according to relevant indicators and effectively realize relevant nursing intervention and drug application for the reasonably prevention of coronary heart disease. Besides, relevant data show that for hypertension patients' vascular elasticity may be weakened due to the influence of abnormal blood pressure. This problem can lead to a trend of greatly weakening the role of vascular elastic reservoir in patients' whole body, which is not conducive to the protection of patients' health. Based on this, the PP and PPI indicators can show a trend of substantial increase. In clinical process, data obtained by ambulatory blood pressure monitoring is more accurate, which can avoid uncertain factors effectively of occasional blood pressure measurement, so as to help medical researchers to further realize reasonable understandings of the patient's blood pressure, which have important significance and value for the follow-up diagnosis and treatment work. In terms of application value, a large number of data show that NPPI and 24hPPI have better predictive value for patients with coronary heart disease and have a good guiding role for maintenance of patients' health. Regarding related problems, through the development and implementation of related research work, a large number of clinical data show that medical 
researchers can effectively analyze the risk of coronary heart disease in patients with hypertension, which has a good promoting significance and guiding role for the reasonable protection of people's health in China.

This study showed that the NPPI, 24hPP and 24hPPI of hypertension patients with coronary heart disease were significantly higher than those of patients with hypertension alone. In the process of clinical diagnosis, the areas under ROC curve of NPPI and 24hPPI are larger than 24hPP, which proves that NPPI and 24hPPI have good application value in predicting coronary heart disease.

To sum up, during the clinical diagnosis of hypertension patients, dynamic pulse pressure and pulse pressure index have a highly relation with the risk of coronary heart disease. Therefore, researchers should actively pay attention to the relevant indicators of patients to lay a solid foundation for the effective protection of patients' health.

\section{References}

1. Gong X, Cai H, Lei Y. Relationship between dynamic pulse pressure and pulse pressure index and coronary heart disease in patients with hypertension. Chinese Journal of Cardiovascular Research 2020; 18(5): 413-417.

2. Gan Y, Wu X, Liu J, et al. Relationship of ankle brachial index and ambulatory pulse pressure with coronary heart disease in elderly hypertensive patients. Chinese Journal of Cardiovascular Review 2008; 6(8): 606-608.

3. Chen J, Zhu J, Lin Y, et al. Relationship between pulse pressure, pulse pressure index and coronary artery disease in 212 elderly patients undergoing coronary angiography (in Chinese). Chinese Journal of Gerontology 2011; 31(10): 1862-1864.

4. Liu H, Liu D. On Correlation between pulse pressure, pulse pressure index and coronary heart disease in elderly patients with hypertension. Journal of Beihua University (Natural Science) 2018; 19(6): 776-779.

5. Wen H, Qi D, Wang X. Application of ankle-brachial index and dynamic pulse pressure in elderly patients with hypertension and diabetes mellitus (in Chinese). Chinese Journal of Gerontology 2009; 29(23): 3113-3114.

6. Feng Y, Shi H, Zhao L. Correlation between ambulatory pulse pressure indexes and coronary artery lesions in patients with hypertension. Chinese Journal of Evidence-Based Cardiovascular Medicine 2013; (4): 369-370.

7. Mo Y, Wu S, Wang Q, et al. Correlation between dynamic pulse pressure index and coronary artery disease in elderly hypertensive patients (in Chinese). Chinese Journal of Gerontology 2006; 26(10): 1351-1352.

8. Mo Y, Wu S, Wang Q, et al. Relationship between pulse pressure index and severity of coronary artery stenosis in elderly hypertensives. Chinese Journal of Geriatric Heart Brain and Vessel Diseases 2006; 8(9): 591-593.

9. You X, Cui W, Ma Y, et al. Correlation between dynamic arteriosclerosis index and carotid intima-media thickness in patients with hypertension and coronary heart disease (in Chinese). Journal of Clinical Internal Medicine 2010; 27(11): 781-782.

10. Li Y, Liu J. Developments in study of effect of pulse pressure and pulse pressure index on target organ damage in essential hypertensive patients. Advances in Cardiovascular Diseases 2008; 29(2): 307-310. 